Articulo Original

\title{
La Norma Humanitaria Esencial de calidad y rendición de cuentas: origen, objetivos y experiencia en Médicos del Mundo
}

\author{
Rocío Poo \\ Técnica de Calidad, Evaluación y Auditoría Interna de Médicos del Mundo; rocio.poo@medicosdelmundo.org; \\ ORCID id: https://orcid.org/0000-0001-6838-3464
}

DOI: https://doi.org/10.37536/RIECS.2020.5.S1.184

Recibido: 26/01/2020; Aceptado: 06/02/2020; Publicado: 14/02/2020

Resumen: En los últimos años una serie de normas, estándares, y certificaciones se han puesto en marcha en el sector de las organizaciones no gubernamentales con el fin de garantizar que desarrollan su trabajo con transparencia y calidad a sus principales grupos de interés (socios, donantes, titulares de derechos y a la sociedad en general). Al mismo tiempo, cada vez más donantes vinculan el cumplimiento de determinadas normas y estándares con la financiación. En este artículo se presenta la Norma Humanitaria Esencial en material de calidad y rendición de cuentas y se justifica la decisión de Médicos del Mundo de analizar si sus acciones en el ámbito de la acción humanitaria cumplen con los compromisos que esta norma establece.

Palabras Clave: Norma Humanitaria Esencial, Acción Humanitaria, Calidad, Evaluación, Rendición de Cuentas, Médicos del Mundo.

\begin{abstract}
In recent years, a series of norms, standards, and certifications have been launched in the non-governmental organizations sector to ensure that they carried out their work with transparency and quality to their main stakeholders (partners, donors, rights holders and society in general). At the same time, more donors link compliance with certain rules and standards with funding. This article presents the Core Humanitarian Standard on Quality and Accountability and justifies the decision of Doctors of the World to assess whether their actions in the field of humanitarian action comply with the commitments established by the standard.
\end{abstract}

Key words: Core Humanitarian Standard, Humanitarian Action, Quality, Evaluation, Accountability, Doctors of the World.

En los últimos años una serie de normas, estándares, y certificaciones se han puesto en marcha en el sector de las organizaciones no gubernamentales con el fin de garantizar que desarrollan su trabajo con transparencia y calidad a sus principales grupos de interés (personas socias, donantes, titulares de derechos y la sociedad en general). Al mismo tiempo, cada vez más donantes vinculan el cumplimiento de determinadas normas y estándares con la financiación.

En este artículo se presenta la Norma Humanitaria Esencial (NHE) en materia de calidad y rendición de cuentas y se justifica por qué Médicos del Mundo ha decidido emprender un proceso de análisis para comprobar si las acciones que implementa en el ámbito de la acción humanitaria cumplen con los compromisos que la norma establece. Por último, se describen los pasos recorridos por Médicos del Mundo en este proceso de certificación y se realiza una valoración del mismo.

Para realizar este artículo se ha analizado la documentación que proporciona la CHS Alliance [1], a través de su página web, para que las organizaciones no gubernamentales puedan verificar su desempeño en materia de calidad y rendición de cuentas, y la normativa establecida por la DG $\mathrm{ECHO}$, para las organizaciones no gubernamentales que quieren acceder a su financiación. Además, 
se han consultado y analizado documentos (manuales, políticas y procedimientos) de Médicos del Mundo.

También se describe el proceso de recogida y análisis de la información realizado por la Unidad de Calidad, Evaluación y Auditoría Interna, con la colaboración de las Unidades de Emergencias y Crisis Complejas del Departamento de Programas Internacionales y el equipo en Ucrania, para valorar en qué medida Médicos del Mundo cumple con los compromisos establecidos por la Norma Humanitaria Esencial.

\section{La Norma Humanitaria Esencial}

La Norma Humanitaria Esencial en materia de calidad y rendición de cuentas surge del trabajo conjunto de una serie de organizaciones del sector humanitario, que tienen en común el objetivo de establecer una autorregulación en el sector de la acción humanitaria para mejorar la rendición de cuentas ante las comunidades y personas afectadas por crisis humanitarias, en el marco de la Joint Standards Initiative (JSI). Estas organizaciones son Humanitarian Accountability Partnership (HAP) International, que había desarrollado las Normas HAP 2010; People in Aid, que había desarrollado el Código de Buenas Prácticas y el Proyecto Esfera, con el establecimiento de la Normas Esenciales.

La Norma Humanitaria Esencial, establece nueve compromisos para las organizaciones y personas involucradas en una respuesta humanitaria. Estos compromisos hacen referencia a la calidad y eficacia de las acciones desarrolladas y a la rendición de cuentas hacia las comunidades y las personas afectadas por una crisis humanitaria sobre estas acciones. La rendición de cuentas establece, como requisito previo, la difusión de estos compromisos entre estas comunidades y personas de modo que, al ser conocidos, estas puedan exigir responsabilidades sobre su cumplimiento.

Cada compromiso, lleva asociado un criterio de calidad, que resume la responsabilidad adquirida por las organizaciones y personas del sector humanitario, y se desglosa en dos componentes, denominados acciones clave y responsabilidades de la organización. Cada componente se operacionaliza mediante una serie de indicadores de desempeño que permiten verificar su cumplimiento por parte de las organizaciones. En las acciones clave se describen las características que las acciones humanitarias implementadas por una organización humanitaria deben comprender, así como aquellos servicios que se deben prestar para cumplir con el compromiso correspondiente. Por otro lado, las responsabilidades de la organización establecen los procesos, políticas, procedimientos o sistemas que las organizaciones han diseñado y puesto en funcionamiento para garantizar que su personal cumple con los compromisos establecidos en la Norma Humanitaria Esencial. En la Tabla 1 , se especifican los compromisos y criterios de calidad.

Tabla I Compromisos y Criterios de Calidad de la Norma Humanitaria Esencial. Fuente: La Norma Humanitaria Esencial en materia de calidad y rendición de cuentas (2015)

\section{COMPROMISO 1 adecuada en relación con sus necesidades. Criterio de Calidad: La respuesta humanitaria es ADECUADA y PERTINENTE.}

Las comunidades y personas afectadas por crisis humanitarias tienen acceso a la

COMPROMISO 2 ayuda que necesitan en el momento adecuado

Criterio de Calidad: La respuesta humanitaria es EFICAZ y proporcionada A TIEMPO.

Las comunidades y personas afectadas por crisis humanitarias no se ven perjudicadas y están más preparadas, son más resilientes y están menos expuestas

COMPROMISO 3 al riesgo como resultado de la acción humanitaria Criterio de Calidad: La respuesta humanitaria FORTALECE LAS CAPACIDADES LOCALES y EVITA CAUSAR EFECTOS NEGATIVOS. 
Criterio de Calidad: La respuesta humanitaria se basa en la comunicación, la PARTICIPACIÓN y la retroalimentación.

Las comunidades y personas afectadas por crisis humanitarias tienen acceso a

COMPROMISO 5 mecanismos seguros y ágiles para gestionar las quejas.

Criterio de Calidad: LAS QUEJAS SON BIEN RECIBIDAS Y GESTIONADAS.

Las comunidades y personas afectadas por crisis humanitarias reciben una ayuda coordinada y complementaria

Criterio de Calidad: La respuesta humanitaria es COORDINADA Y COMPLEMENTARIA

Las comunidades y personas afectadas por crisis humanitarias pueden esperar que se les brinde una mejor asistencia, ya que las organizaciones aprenden de la

COMPROMISO 7 experiencia y la reflexión.

Criterio de Calidad: Los actores humanitarios están en un proceso de APRENDIZAJE y MEJORA CONSTANTE.

Las comunidades y personas afectadas por crisis humanitarias reciben la ayuda que necesitan por parte del personal y voluntarios competentes gestionados de forma

COMPROMISO 8 adecuada

Criterio de Calidad: El PERSONAL CUENTA CON APOYO PARA HACER SU TRABAJO con eficacia y RECIBE UN TRATO JUSTO Y EQUITATIVO.

Las comunidades y personas afectadas por crisis humanitarias pueden esperar que las organizaciones que les prestan asistencia gestionen los recursos de forma

COMPROMISO 9 efectiva, eficaz y ética.

Criterio de Calidad: Los RECURSOS SE GESTIONAN Y USAN DE FORMA RESPONSABLE PARA LOS FINES PREVISTO

La NHE se aplica en todas las fases del ciclo de gestión de la acción humanitaria. Si bien cada compromiso no se corresponde con una fase concreta, algunos compromisos son más relevantes para alguna fase en concreto, mientras que otros, más relacionados con la comunicación y rendición de cuentas, afectan a todo el ciclo de intervención. En la Tabla II se especifican los compromisos y criterios de calidad a lo largo de todo el ciclo.

Tabla II Compromisos y Criterios de Calidad de la Norma Humanitaria Esencial. Fuente: Elaboración propia a partir de la NHE

\begin{tabular}{|c|c|}
\hline $\begin{array}{l}\text { FASE DEL CICLO DE GESTIÓN DE } \\
\text { UNA INTERVENCIÓN }\end{array}$ & $\begin{array}{c}\text { COMPROMISOS DE LA NHE } \\
\text { SOBRE LAS INTERVENCIONES HUMANITARIAS }\end{array}$ \\
\hline IDENTIFICACIÓN & Adecuada y pertinente \\
\hline PLANIFICACIÓN/DISEÑO & $\begin{array}{l}\text { Fortalece las capacidades locales y evita causar efectos } \\
\text { negativos } \\
\text { Es eficaz y proporcionada a tiempo. } \\
\text { Es coordinada y complementaria }\end{array}$ \\
\hline IMPLEMENTACIÓN & $\begin{array}{l}\text { Los recursos se gestionan y usan de forma responsable para } \\
\text { los fines previsto } \\
\text { El personal cuenta con apoyo para hacer su trabajo con eficacia } \\
\text { y recibe un trato justo y equitativo. } \\
\text { La respuesta humanitaria es eficaz y proporcionada a tiempo. } \\
\text { Es coordinada y complementaria } \\
\text { Las quejas son bien recibidas y gestionadas. }\end{array}$ \\
\hline SEGUIMIENTO Y EVALUACIÓN & $\begin{array}{l}\text { los actores humanitarios están en un proceso de aprendizaje y } \\
\text { mejora constante } \\
\text { Las quejas son bien recibidas y gestionadas. }\end{array}$ \\
\hline
\end{tabular}




\section{El Proyecto Esfera y la Norma Humanitaria Esencial}

El Proyecto Esfera, denominado en la actualidad Esfera, se pone en marcha, en 1997, por un grupo de organizaciones no gubernamentales con el doble objetivo de mejorar la calidad y la rendición de cuentas de las actuaciones en el ámbito de la acción humanitaria. El Manual Esfera [2] constituye una importante herramienta para las organizaciones que trabajan en este ámbito ya que recoge los principios y normas para la respuesta humanitaria más reconocidos en el sector a nivel internacional.

Los principios y fundamentos de las normas Esfera están compuesto por tres elementos: la Carta Humanitaria que "expresa la convicción común de los actores humanitarios de que todas las personas afectadas por una crisis tienen derecho a recibir protección y asistencia. Este derecho garantiza las condiciones básicas para vivir con dignidad" y proporciona el fundamento ético y jurídico para todos los elementos que conforman Esfera; los cuatro Principios de Protección que fundamentan la respuesta humanitaria en la práctica; y las seis Normas Esenciales que establecen los procesos y enfoques (respuesta humanitaria centrada en las personas; coordinación y colaboración; evaluación; diseño y respuesta; desempeño, transparencia y aprendizaje y desempeño de los trabajadores humanitarios) que son fundamentales para que la intervención humanitaria sea eficaz.

En noviembre de 2018, coincidiendo con el vigésimo aniversario del Manual Esfera, las Normas Esenciales se sustituyen por la Norma Humanitaria Esencial en lo que supone un avance en la simplificación y unificación de los procesos de calidad en el ámbito humanitario. Por lo que, en la actualidad, aquellas organizaciones que establezcan que sus actuaciones en el ámbito humanitario deben cumplir con los principios y fundamentos establecidos en el Manual Esfera, deben seguir los nueve compromisos que conforman la Norma Humanitaria Esencial.

\section{La Norma Humanitaria Esencial y los criterios de evaluación del CAD}

La NHE es de aplicación a las organizaciones y personal que prestan asistencia a las comunidades y personas afectadas por crisis humanitarias, mientras que los seis criterios de evaluación (eficacia, eficiencia, pertinencia, sostenibilidad, impacto y coherencia) del CAD de la OCDE están enfocados a las intervenciones de desarrollo. Sin embargo, ambos sistemas guardan una estrecha relación, como se puede ver en la Tabla III, donde se han clasificado los criterios de calidad de la NHE según el criterio de evaluación del CAD con el que se corresponden.

Tabla III Relación entre los Criterios de Evaluación del CAD y los Compromisos de la Norma Humanitaria Esencial Fuente: Elaboración propia a partir de los Criterios de Evaluación del CAD y la NHE

\begin{tabular}{|c|c|}
\hline $\begin{array}{l}\text { CRITERIOS DE } \\
\text { EVALUACIÓN DEL } \\
\text { CAD }\end{array}$ & $\begin{array}{l}\text { CRITERIOS DE CALIDAD DE LA NORMA HUMANITARIA } \\
\text { ESENCIAL }\end{array}$ \\
\hline EFICACIA & Compromiso 2: La respuesta humanitaria es EFICAZ \\
\hline EFICIENCIA & $\begin{array}{l}\text { Compromiso 2: La respuesta humanitaria es proporcionada A TIEMPO } \\
\text { Compromiso 9: Los RECURSOS SE GESTIONAN Y USAN DE FORMA } \\
\text { RESPONSABLE PARA LOS FINES PREVISTO }\end{array}$ \\
\hline PERTINENCIA & $\begin{array}{l}\text { Compromiso 1: La respuesta humanitaria es ADECUADA y PERTINENTE. } \\
\text { Compromiso 4: La respuesta humanitaria se basa en la comunicación, la } \\
\text { PARTICIPACIÓN y la retroalimentación }\end{array}$ \\
\hline COHERENCIA & $\begin{array}{lrllll}\text { Compromiso 6: La respuesta humanitaria es COORDINADA } & \text { Y } \\
\text { COMPLEMENTARIA } & & & & & \\
\end{array}$ \\
\hline SOSTENIBILIDAD & $\begin{array}{l}\text { Compromiso 3: La respuesta humanitaria FORTALECE LAS } \\
\text { CAPACIDADES LOCALES } \\
\text { Compromiso 4: La respuesta humanitaria se basa en la PARTICIPACIÓN }\end{array}$ \\
\hline IMPACTO & $\begin{array}{l}\text { Compromiso 3: La respuesta humanitaria EVITA CAUSAR EFECTOS } \\
\text { NEGATIVOS }\end{array}$ \\
\hline
\end{tabular}


Como resultado de este análisis encontramos que todos los criterios de evaluación del CAD se corresponden con al menos un compromiso de la NHE. Luego las acciones que se implementan en el ámbito humanitario y que cumplen con los compromisos establecidos por la NHE también serían intervenciones de calidad según el CAD. Pero, además, la NHE establece tres criterios de calidad adicionales que no están contemplados en ninguno de los criterios de evaluación del CAD:

- Criterio de calidad 5. Las quejas son bien recibidas y gestionadas

- Criterio de calidad 7. Los actores humanitarios están en un proceso de aprendizaje y mejora constante

- Criterio de calidad 8. El personal cuenta con apoyo para hacer su trabajo con eficacia y recibe un trato justo y equitativo.

Estos criterios adicionales están relacionados con el valor que, desde la NHE, se da a las personas y comunidades afectadas por las crisis, ya que las sitúa en el centro de la acción humanitaria. Si los criterios de calidad del CAD son elaborados por los principales donantes y, por lo tanto, sitúan a estos como principales destinatarios de la rendición de cuentas, la NHE establece que son las personas y comunidades afectadas por las crisis a quienes hay que rendir cuentas y cuyas opiniones deben ser tenidas en consideración.

Por otro lado, si bien los criterios de evaluación del CAD tienen la función establecer las normas que deben cumplir las intervenciones de desarrollo para ser de calidad, la NHE va más allá, al instaurar la posibilidad de que las organizaciones que trabajan en el ámbito humanitario se certifiquen. De este modo, se establecen no solo unos lineamientos sobre buenas prácticas en el sector, sino que también proporciona un poco para la diferenciación, basada en una certificación independiente realizada por auditores aprobados para ello, entre aquellas organizaciones que cumplen con la NHE y aquellas que no.

\section{Por qué Médicos del Mundo ha decidido implantar la Norma Humanitaria Esencial}

La decisión de implantar la NHE en Médicos del Mundo se fundamenta en dos tipos criterios. Por un lado, en el cumplimiento de los principios, normas y políticas que se ha autoimpuesto la organización, y que se establecen en una serie de documentos, y, por otro lado, en los requerimientos establecidos por nuestros financiadores ${ }^{1}$.

En relación con el primer criterio, la Política de Acción Humanitaria, aprobada por la Junta Directiva en mayo de 2010, establece que la Acción Humanitaria de Médicos del Mundo se fundamenta1 en el Proyecto Esfera, que comprende, como se ha señalado, la Carta Humanitaria, los Principios y las Normas; Además, el Manual de Acción Humanitaria de Médicos del Mundo [3, 4] establece que, en el marco de las actuaciones que se lleven a cabo en este ámbito, se deben seguir "las orientaciones técnico-metodológicas y los estándares de calidad adecuados para el cumplimiento de los requerimientos exigidos (normas de calidad internacionales, buen reporting y rendición de cuentas, información/visibilidad)"; Por otro lado, el Emergency Operational Framework de la Red Internacional de Médicos del Mundo, aprobado en octubre de 2017, indica que Médicos del Mundo "se compromete a seguir los estándares humanitarios internacionalmente aceptados (por ejemplo, Esfera)". En este sentido, al incluirse, en noviembre de 2018, la NHE como parte de Esfera surge en Médicos del Mundo la necesidad de realizar un análisis del cumplimiento de los requerimientos establecidos por la NHE.

En cuanto al segundo criterio, hay que señalar que Médicos del Mundo tiene firmado un Acuerdo Marco de Asociación (Framework Partnership Agreement-FPA) [5] con la Dirección General de Protección Civil y Operaciones de Ayuda Humanitaria Europeas (DG ECHO, por sus siglas en inglés) de la Comisión Europea. Este Acuerdo Marco de Asociación identifica los derechos y obligaciones generales de las partes y especifica las normas que rigen las acciones de ayuda humanitaria

\footnotetext{
${ }^{1}$ También se fundamenta en la Política de Inclusión de una Perspectiva de Género en la Acción Humanitaria del Comité Permanente entre Organismos de Naciones Unidas (IASC) (1999) y en el Código de Conducta para organizaciones humanitarias en casos de desastre (1994).
} 
financiadas por la UE. El actual FPA² establece una serie de obligaciones para Médicos del Mundo recogidas también en los compromisos derivados de la NHE. En la siguiente tabla (Tabla IV) se muestran las obligaciones derivadas del cumplimiento del FPA que, a su vez, se corresponden con compromisos establecidos por la NHE:

Tabla IV Relación entre la Norma Humanitaria Esencial y las obligaciones derivadas del cumplimiento del Framework Partnership Agreement-FPA. Fuente: Elaboración propia a partir de la NHE y el FPA de DG ECHO

\begin{tabular}{|c|c|}
\hline FPA (2014) & CHS \\
\hline \multicolumn{2}{|l|}{ Artículo 2. Principios e Implementación del FPA } \\
\hline $\begin{array}{l}\text { - Transparencia y responsabilidad ante todos los interesados, } \\
\text { incluidos los beneficiarios de la ayuda }\end{array}$ & Compromiso 4 \\
\hline $\begin{array}{l}\text { - Promoción de una cultura de aprendizaje basada en la } \\
\text { evaluación de acciones y en compartir y difundir las mejores } \\
\text { prácticas y lecciones aprendidas }\end{array}$ & Compromiso 7 \\
\hline \multicolumn{2}{|l|}{$\begin{array}{l}\text { Artículo 5. Mínimos estándares para la preparación e implantación } \\
\text { de las acciones }\end{array}$} \\
\hline $\begin{array}{l}\text { - Asignar fondos de acuerdo con las evaluaciones de } \\
\text { necesidades }\end{array}$ & Compromiso 1 \\
\hline $\begin{array}{l}\text { - Promover la participación de los beneficiarios en el } \\
\text { establecimiento, implementación y evaluación de acciones; }\end{array}$ & Compromiso 4 \\
\hline $\begin{array}{l}\text { - Sustentar las acciones en las capacidades locales, respetando } \\
\text { la cultura, la estructura y las costumbres de las } \\
\text { comunidades y de los países donde se llevan a cabo las } \\
\text { acciones }\end{array}$ & $\begin{array}{l}\text { Compromiso } 3 \\
\text { Compromiso } 1 \\
\text { Compromiso } 4\end{array}$ \\
\hline $\begin{array}{l}\text { - Asegurar 'tolerancia cero' con la explotación y el abuso } \\
\text { sexual a través de prevención, informes y respuestas } \\
\text { efectivas y coordinadas }\end{array}$ & $\begin{array}{l}\text { Compromiso } 5 \\
\text { Compromiso } 8\end{array}$ \\
\hline $\begin{array}{l}\text { - Contribuir al fortalecimiento de las capacidades de las } \\
\text { comunidades afectadas, a fin de prevenir, prepararse, } \\
\text { reducir y responder a futuras crisis humanitarias }\end{array}$ & Compromiso 3 \\
\hline $\begin{array}{l}\text { - Provisión de condiciones laborales justas para trabajadores } \\
\text { humanitarios, voluntarios o asalariados, con especial } \\
\text { atención a su seguridad en el campo y, en la medida de lo } \\
\text { posible, a su desarrollo profesional; }\end{array}$ & Compromiso 8 \\
\hline $\begin{array}{l}\text { - Un sistema de control interno eficaz y eficiente para la } \\
\text { gestión de acciones que incluye el respeto de los valores } \\
\text { éticos y humanitarios, la segregación efectiva de funciones } \\
\text { y los mecanismos adecuados de gestión de riesgos, la } \\
\text { identificación de riesgos y las respuestas de riesgo } \\
\text { apropiadas }\end{array}$ & $\begin{array}{l}\text { Compromiso } 9 \\
\text { Compromiso } 2 \\
\text { Compromiso } 3\end{array}$ \\
\hline - $\quad$ Un sistema contable preciso, completo y oportuno & Compromiso 9 \\
\hline $\begin{array}{l}\text { - La disponibilidad de información relevante a su debido } \\
\text { tiempo para una gestión adecuada }\end{array}$ & Compromiso 2 \\
\hline
\end{tabular}

En estos momentos Médicos del Mundo ha empezado el proceso para la firma de un nuevo FPA con la DG ECHO que entraría en vigor en enero de 2021. Para ello Médicos del Mundo debe proporcionar a la CE una garantía de que cumple con el conjunto de criterios establecidos por ECHO, basados en el Reglamento de Ayuda Humanitaria, el Reglamento Financiero y las mejores prácticas internacionales, que demuestran que: es una organización transparente; cumple con los más altos

${ }^{2} \mathrm{El}$ actual FPA entro en vigor el 1 de enero de 2014 y tiene validez hasta el 31 de diciembre de 2020. 
estándares éticos; despliega los medios adecuados para abordar las irregularidades, el fraude, la corrupción y la mala conducta de cualquier tipo; cumple con los principios humanitarios; tiene una capacidad operativa adecuada y tiene un sólido sistema de control interno. En este nuevo FPA la DG ECHO establece específicamente como mejores prácticas internacionales el Manual Esfera y la Norma Humanitaria Esencial de Calidad y Rendición de Cuentas.

\section{El análisis de la aplicación de la Norma Humanitaria Esencial en Médicos del Mundo}

Médicos del Mundo se propuso en 2019 empezar el proceso de análisis del cumplimiento de la NHE realizando una Autoevaluación. La Autoevaluación es la modalidad propuesta por la CHS Alliance para aquellas organizaciones que quieren conocer en qué medida están aplicando los compromisos de la NHE. Esta autoevaluación permite identificar puntos de mejora y preparar a la organización para una posible certificación. La CHS Alliance proporciona unos manuales de orientación y un modelo de informe para que la organización pueda de forma interna autoevaluarse.

El proceso de Autoevaluación de la NHE en Médicos del Mundo ha sido liderado por la Unidad de Calidad, Auditoría Interna y Evaluación, y respaldado por la Junta Directiva, tal como establecen los manuales de la CHS. Este proceso ha comprendido los siguientes pasos:

- La revisión de políticas, estrategias, manuales y procedimientos de Médicos del Mundo;

- La realización de diferentes grupos focales y entrevistas con el personal de la Unidades de Emergencias y Crisis Complejas, del Departamento de Desarrollo de Personas, del Departamento de Comunicación y del Departamento Económico Financiero de la sede central de Médicos del Mundo;

- Para realizar el trabajo de campo se escogió Ucrania por ser un país representativo del trabajo de la organización. La Unidad de Calidad se desplazó a Ucrania donde se realizaron entrevistas y grupos focales con el personal de Médicos del Mundo en este país y con las personas afectadas por la crisis humanitaria, así como con sus representantes en los servicios públicos de salud; y

- La realización de un informe de autoevaluación en el que se describe en qué medida la organización cumple con los indicadores establecidos en los nueve compromisos y las evidencias recogidas durante el proceso que justifican lo indicado en el informe.

A partir de la valoración que la CHS Alliance haga del Informe de Autoevaluación de Médicos del Mundo se identificarán buenas prácticas y ámbitos de mejora. Por lo que el siguiente paso en este proceso consistirá en la elaboración e implementación de un Plan de Mejora en función de las debilidades encontradas.

\section{Conclusiones: valoración de la experiencia en Médicos del Mundo}

Como se ha podido comprobar en este artículo el proceso de autoevaluación del cumplimiento de la NHE de calidad y rendición de cuentas ha sido complejo y ha implicado la inversión de recursos financieros y del tiempo del personal que trabaja en distintos departamentos, tanto en la sede central como en el terreno, de Médicos del Mundo. Sin embargo, este la realización de este análisis era ineludible al formar parte tanto de los requerimientos internos que Médicos del Mundo se ha autoimpuesto como, al coincidir, con los requerimientos establecidos por financiadores estratégicos para la organización.

Médicos del Mundo al adoptar la NHE asume un mayor compromiso de eficacia y transparencia de sus actuaciones con las personas afectadas por las crisis humanitarias, incorporando criterios de calidad no contemplados en otros sistemas de evaluación como pueden ser los criterios de evaluación del CAD de la OCDE.

A partir de ahora, el reto para Médicos del Mundo es no quedarse en el proceso de revisión e impulsar planes de mejora fundamentados en los principales hallazgos. 
Agradecimientos: Se agradece al personal de Médicos del Mundo (Unidades de Emergencias y Crisis Complejas del Departamento de Programas Internacionales, Departamento de Desarrollo de Personas, Departamento de Comunicación, Departamento Económico Financiero y de Ucrania) el trabajo desarrollado para analizar el cumplimiento de la NHE. También a Pablo Hernández, Coordinador de Calidad, Evaluación y Auditoría Interna, sus aportaciones y la revisión del documento.

Conflictos de Intereses: "los autores no declaran conflicto de intereses".

\author{
Abreviaturas \\ Las siguientes abreviaturas son usadas en este manuscrito: \\ CAD: Comité de Ayuda al Desarrollo \\ CE: Comisión Europea \\ DG ECHO: Dirección General de Protección Civil y Operaciones de Ayuda Humanitaria Europeas \\ FPA: Framework Partnership Agreement \\ NHE: Norma Humanitaria Esencial \\ OCDE: Organización para la Cooperación y el Desarrollo Económicos
}

\title{
Referencias Bibliográficas
}

1. CHS Alliance, Group URD y el Proyecto Esfera. La Norma Humanitaria Esencial en materia de Calidad y Rendición de Cuentas. 2015. Disponible en:

https://corehumanitarianstandard.org/files/files/Core\%20Humanitarian\%20Standard\%20-

\%20Spanish.pdf

2. Sphere. El Manual Esfera. Carta Humanitaria y normas mínimas para la respuesta humanitaria. 2018. Disponible en: https://spherestandards.org/wp-content/uploads/El-manual-Esfera-2018-ES.pdf

3. International Network of MdM. Emergency Operational Framework. 2017.

4. Médicos del Mundo. Manual de Acción Humanitaria de Médicos del Mundo. 2013

5. Model of the Framework Partnership Agreement with Humanitarian Organisations FPA 01/01/2014.

Disponible en: https://ec.europa.eu/echo/files/partners/humanitarian_aid/fpa/2014/FPA_NGO_en.pdf

(C) 2020 por los autores; Esta obra está sujeta a la licencia de Reconocimiento 4.0

Internacional de Creative Commons. Para ver una copia de esta licencia, visite http://creativecommons.org/licenses/by-nc-nd/4.0/. 